\title{
Virtual Robot Teaching Based on Motion Analysis and Hand Manipulability for Multi-Fingered Robot*
}

\author{
Haruhisa KAWASAKI**, Tomohiro FURUKAWA**, Satoshi UEKI*** \\ and Tetsuya MOURI** \\ ** Department of Human Information Engineering, Gifu University \\ 1-1 Yanagito, Gifu, Japan \\ *** Virtual System Laboratory, Gifu University \\ 1-1 Yanagito, Gifu, Japan
}

\begin{abstract}
A virtual robot teaching system, consisting of human demonstration and motion-intention analysis in a virtual reality environment, is an advanced form of automatic programming for multi-fingered robots. We propose a new segmentation method to analyze human motion data and a virtual teaching system based on hand manipulability, in which the position and orientation of the robot hand are determined so as to maximize the robot hand's manipulability. In the segmentation method, human motion data consisting of contact points, grasp force, hand and object position, and the like are segmented into plural primitive motions and the type of task is analyzed based on the sequence of the primitive motions. A trial assembly task using a humanoid robot hand named Gifu Hand III is shown to demonstrate the effectiveness of the proposed method.
\end{abstract}

Key words: Robot, Teaching, Virtual Reality, Robot Hand

\section{Introduction}

Humanoid robots are expected to relieve humans of various tasks, such as high-mix low-volume production and assembly tasks. Robot programming technologies such as robot language and teaching-playback (e.g., Asada ${ }^{(1)}$ ) have been developed, though it is difficult to apply these technologies to multi-fingered robots because they require the instruction of both motions and forces at many points simultaneously.

Recently, several techniques that directly use human motion measurements as robot teaching data have been proposed for automatic programming: teaching by showing (Kuniyoshi, et al. ${ }^{(2)}$ ), assembly plan from observation (K. Ikeuch et al. ${ }^{(3)}$, Kang et al. ${ }^{(4)}$ ), and gesture-based programming (Voyles, et al. ${ }^{(5)}$ ). Task programming based on the observation of human operation is viable for humanoid robots because it is not necessary to describe motions and forces explicitly for the robot to accomplish a task.

Direct teaching that involves remote robot operation presents two difficulties. One results from the communication time lag that arises when the robot is located apart from the operator, which can make the remote-robot system unstable. The other is the fact that the operator undergoes continual stress because any mistake on his or her part is immediately reflected in the robot motions and may result in a fatal accident. Robot teaching in a virtual reality (VR) environment can overcome these problems. Takahashi et al. ${ }^{(6)}$ have presented a robot teaching method using a data glove to measure the finger motion of the human in a VR environment; Yoshikawa et al. ${ }^{(7)}$ presented a haptic simulator; Kaiser et al. ${ }^{(8)}$ presented an analysis of human skills; Sato et al. ${ }^{(9)}$ presented an understanding of human intention;

*Received 28 Aug., 2008 (No. 08-0622) [DOI: 10.1299/jamdsm.3.1] 
and Tsuda et al. ${ }^{(10)}$ presented a method for generating an assembly-task model from human demonstration. Most of these researches do not handle the virtual force generated in the VR environment as robot teaching data.

Our group has presented a concept of virtual robot teaching for multi-fingered robots (11)-(12), in which the virtual forces at contact points are utilized. Effectiveness of the virtual robot teaching has been shown only for a single task. Computer graphics can generate and display two types of virtual hand model: that of a human-hand model, and that of a robot-hand model. The virtual hand model mimics the motion of the human hand or the robot hand and is displayed to the human. The human performs the virtual task while observing the virtual hand. The type of virtual hand model displayed on-screen should be selected carefully to optimize teaching efficiency ${ }^{(13)}$. None of the previous reports have discussed the comparative effectiveness of virtual hand models generated by computer graphics. The robot hand can directly execute teaching data based on a robot-hand model generated by computer graphics, but a human being will have difficulty handling a virtual hand based on the robot-hand model because the kinematics and movable ranges are not the same. On the other hand, a human will find it easy to handle a virtual hand based on a human hand model. The robot, however, cannot grasp an object by using the teaching data because the geometrical form and motional function of the robot hand is not the same as that of the human hand. Kang et al. ${ }^{(14)}$ presented a mapping from human grasps to robot grasps at the functional and physical steps, and Rohling et al., ${ }^{(15)}$ presented an optimized fingertip mapping that minimizes the difference between human-robot fingertip position and orientation, but these did not take into the manipulability of robot hand into consideration. Hand manipulability is a key measure for stable and robust robot grasps. But, the optimum solution, which maximizes the hand manipulability, is computed by the steepest descent method and sometimes is not obtained for the initial hand position given by virtual teaching because of unsolvability of the inverse kinematics problem.

This paper first presents a new segmentation method for processing human motion data including plural tasks. In this method, segmentation of motion, task recognition, and re-segmentation of motion are executed sequentially, and human motion data consisting of contact points, grasped force, hand and object positions, and the like are segmented into plural primitive motions based on a segmentation tree: the type of task is analyzed based on the acquired sequence of primitive motions, and re-segmentation of the motion is executed sequentially. In this method, the segmentation tree is additive for any new primitive motion that is required as part of performing a new task. Secondly, in order to be able to map teaching data based on the human-hand model to teaching data for a robot hand, a virtual teaching method for multi-fingered robots based on a combination of scaling of the virtual hand model to the size of the robot hand and hand manipulability is presented. In this method, the position and orientation of the robot hand are determined so as to maximize the manipulability of the robot hand, on the condition that the robot grasps the object at the object's teaching contact point. This approach makes the virtual teaching system user-friendly. The experimental results of performing an assembly task using a humanoid robot hand named Gifu Hand III ${ }^{(16)}$ are shown.

\section{Virtual Robot Teaching}

\subsection{Scheme of Virtual Robot Teaching}

A conceptual scheme of the virtual robot teaching system (Kawasaki et al. ${ }^{(11)-(12)}$ ) is shown in Fig. 1. The system consists of a VR robot teaching system and a robot system that is located in a remote place. In the former system, the human in a VR environment carries out various tasks employing a virtual object. From the series of human motions and virtual forces acting on the human's fingers, the motion intention of the human is analyzed. Based on the motion intention analysis, a series of robot commands, which include the desired 


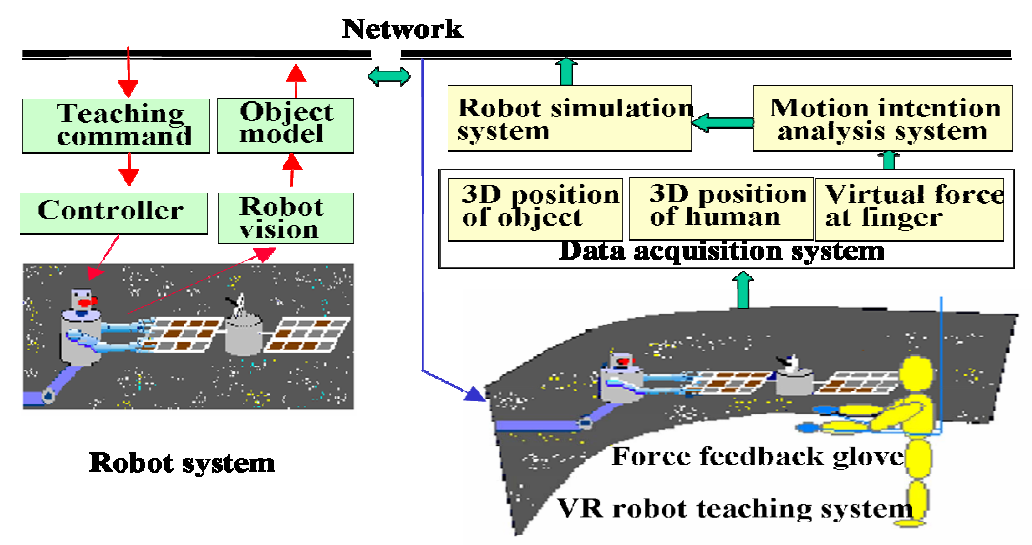

Fig. 1 Conceptual figure of virtual robot teaching

trajectories, the desired grasping forces, and the desired contact points, are generated for the object coordinate frame. The robot commands are tested in the robot simulation system and are then sent to the remote robot system. In the robot system, the robot works according to the robot commands. The robot system can absorb a slight geometrical difference between the virtual space and the real space, because the robot obeys commands relative to the object coordinate frame.

This scheme has two advantages. The first is that the communication time delay has no effect, since the robot commands are generated from the off-line motion analysis. The second is that the human does not undergo mental stress, since any inadvertent human error can be compensated for.

\subsection{Robot with Gifu Hand III}

We consider a multi-fingered hand robot equipped with the Gifu Hand III developed by our group ${ }^{(16)}$. The shape and mechanism of Gifu Hand III are designed to resemble those of the human hand as shown in Fig. 2. That is, it has a thumb and 4 fingers, the thumb has 4 joints with 4 degree of freedoms (DOF), and each finger has 4 joints with 3 DOF. All servomotors are mounted in the hand frame. A 6-axis force sensor can be attached to each fingertip, and a distributed tactile sensor with 859 detecting points can be mounted on the surfaces of the palm and fingers. Since the Gifu Hand III is designed such that not only the shape but also the mechanism are very similar to those of the human hand, as long as the shape of the object is simple and the size is manageable, most of the measured human motion data can be applied directly to the robot command, assuming two conditions are met: first, the object must have a simple shape and be of a manageable size; second, the virtual hand in the VR environment must be scaled to the Gifu Hand III in real space.

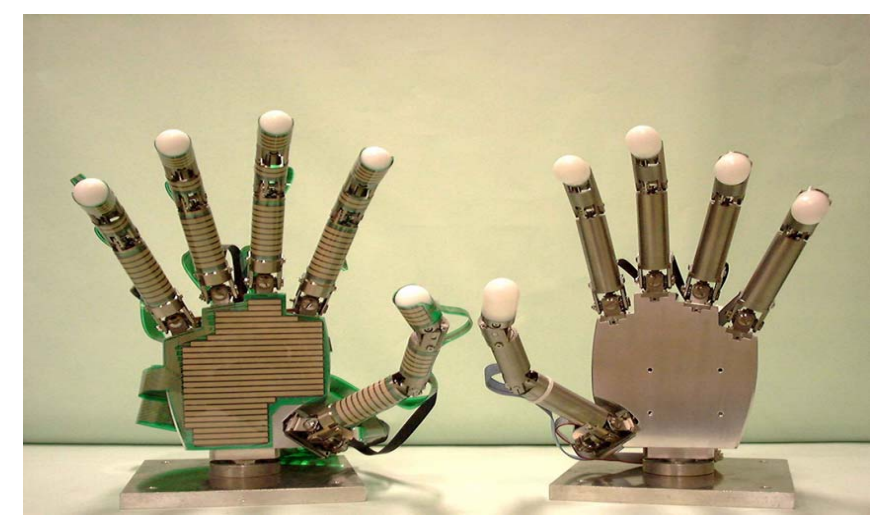

Fig. 2 Humanoid robot hand: Gifu Hand III 


\subsection{Force Feedback Glove}

The VR environment must be realistic such that the human operator feels as if he or she is manipulating real objects in the real world. Force information, particularly with regard to tactility, is therefore crucial. The force as well as the visual effect should be presented appropriately to the human. In our system, the forces generated during the grasping of a virtual object are displayed to the human hand using a developed force feedback glove (FFG) ${ }^{(17)}$, as shown in Fig. 3. The operator puts on the FFG. The FFG consists of a force feedback mechanism and a data glove (Cyber Glove, Virtual Technologies Co. ${ }^{(18)}$ ), which is used to measure the joint angle of the human finger. The force feedback mechanism has 5 servomotors located on the backside of the hand to represent fingertip force. The torque driven by the servomotor is transmitted to the human fingers through the wire rope. The force at the finger is measured by a force sensor, which is a pressure-sensitive conductive elastomer sensor. The human can feel the forces at the fingertips. The resolution and maximum of the grasping force generated by the FFG are about $0.3[\mathrm{~N}]$ and $6[\mathrm{~N}]$, respectively. The force mechanism has also 12 vibrating motors located on the finger cushion of the fingers and the palm to present touch timing. The human can feel the touch timing exactly at 2 points on each digit, 2 points on the thumb, and 2 points on the palm, for a total of 12 points on the hand. Using this device, the virtual forces are communicated to the human. In order to use the FFG, exact information is required on the geometrical relation between the virtual hand and the virtual object, the contact points, and the contact forces. We supposed that the geometry of the object is known or measured, and contact points and contact force are computed using the virtual hand model, the virtual object model, and the fingertip positions of the operator.

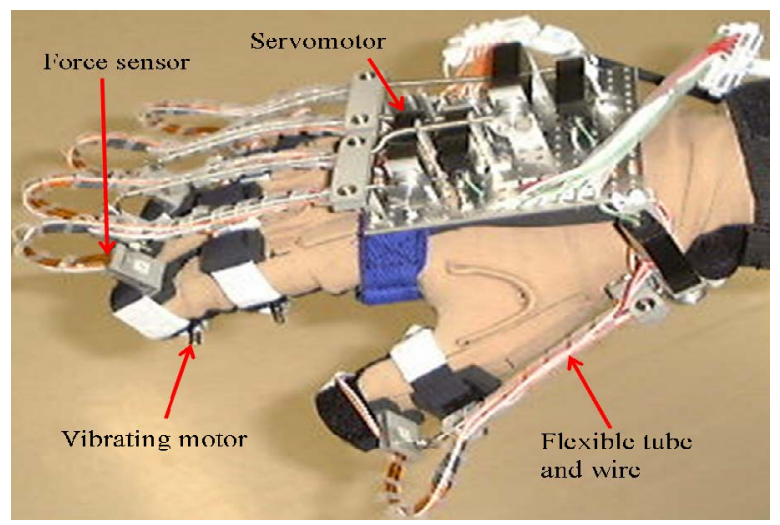

Fig. 3 Force feedback glove

\subsection{Motion segmentation}

It is difficult to analyze the intention of human motion solely by measuring the motion, given the wide range of possible intentions. However, in cases of simple tasks such as the pick-and-place and peg-in-hole tasks in assembly work, the motion intended by the human appears in a series of motion trajectories. In the motion intention analysis system, a task is segmented into primitive motions, which cumulatively form the basis for analyzing the motion intention.

Let us consider the segmentation of a pick-and-place task. Kang et al. ${ }^{(14)}$ presented a technique for segmenting the motions involved in human grasps in the real world, but this technique did not consider virtual forces. In our approach ${ }^{(1)}$, the virtual forces at multiple fingers were used as a key factor in the motion intention analysis. In this case, we assumed that the task handled in motion intention analysis was known. Assembly work consists of plural tasks such as pick-and-place tasks, peg-in-hole tasks, peg-pullout-from-hole tasks, and so on. Hence, the motion intention analysis system should have the ability to recognize the type of tasks from human motion data. A new segmentation method, in which 


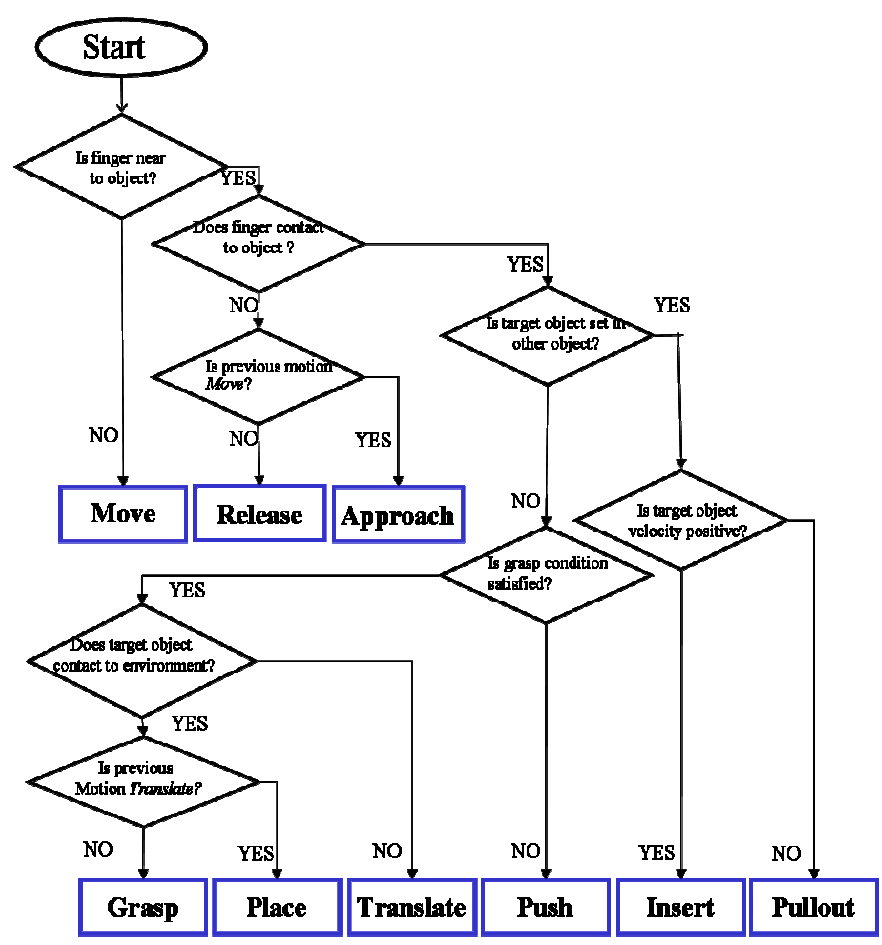

Fig. 4 Flowchart of segmentation of motion data

segmentation of motion, task recognition, and re-segmentation of motion are executed sequentially, is proposed to realize this function.

A flowchart of the segmentation of motion data is shown in Fig. 4. In this segmentation tree, three tasks, that is, a pick-and-place task, peg-in-hole task, and peg-pullout-from-hole task, are considered. We assumed that the human motion to execute these tasks consists of 9 primitive motions as follows: Move, Approach, Grasp, Translate, Push, Insert, Pullout, Release, and Place. Move is a section in which only the operator's hand moves at a point distant from a virtual object. Approach is a section in which the operator's fingertip is located near a virtual object, but does not touch it, and the previous motion is Move. Grasp is a section in which the finger contacts an object located on a base or another object, the grasp condition is satisfied and the previous motion is not Translate. The grasp condition means that the virtual force which is generated by the interference between the finger and virtual object is greater than a specified value. This grasp is a fingertip grasp ${ }^{(19)}$. Translate is a section in which the object moves with the hand and fingers as one unit; that is, the virtual object departs from the base or another object and the grasp condition is satisfied. Place is a section in which the object contacts the environment and the previous motion is Translate. In human operation, it is difficult to separate the Translate segment and Place segment exactly, and indeed the operator does not feel that these can be separated. We assumed that the start point of the Place segment is the moment that the virtual object contacts the environment first. Release is a section in which the fingertip leaves the object and the previous motion is not Move. Hence, the start point of Release segment is the moment that one of the fingertips leaves the object. Push is a section in which the object is touched by the hand, but the grasp condition is not satisfied. In this motion, the object is translated to a point. Insert is a section in which a finger contacts a target object that is set inside another object, and the target object moves in a direction toward the other object. Pullout is a section in which finger contact a target object that is set inside another object, and the target object moves in a direction away from the other object.

To segment the motion data, three coordinate frames are utilized: the reference coordinate frame, the origin of which is fixed in the task space; the object coordinate frame fixed in the object; and the hand coordinate frame fixed in the hand. The following 4 


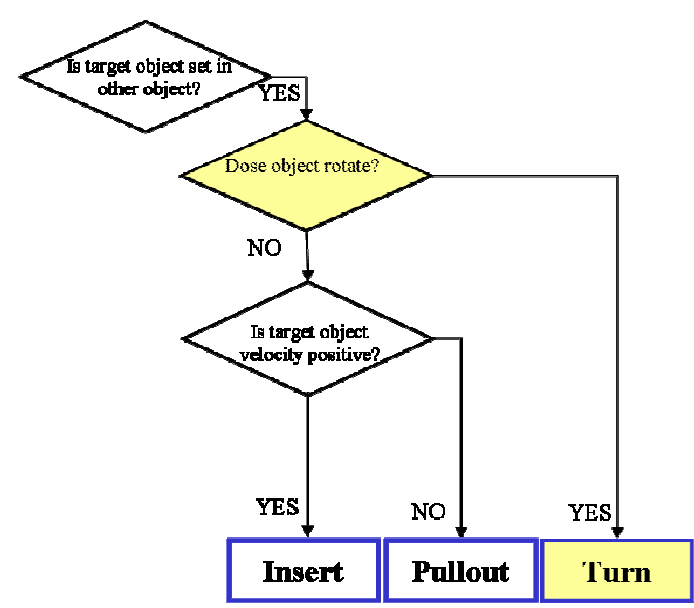

Fig. 5 Addition of Turn in the segmentation

parameters are measured as the motion data: an object position with respect to the reference coordinate frame ${ }^{\text {ref }} \boldsymbol{p}_{\text {object, }}$, an $i$-th fingertip position with respect to the object coordinate

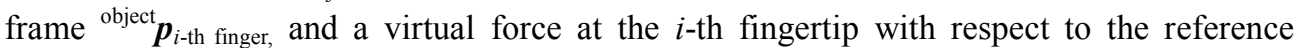
coordinate frame ${ }^{\text {ref }} \boldsymbol{f}_{i \text {-th finger. Moreover, the hand velocity }}{ }^{\text {object }} \boldsymbol{v}_{\text {hand }}$, the object velocity ${ }^{\text {ref }} \boldsymbol{v}_{\text {object }}$, the index of the grasp space of hand ${ }^{\text {object }} \mathrm{P}_{\text {finger }}\left(=\sum_{i=1}^{5}\|\|^{\text {object }} \boldsymbol{p}_{i \text {-th finger }} \|\right)$, and the sum of fingertip forces in the direction of gravitation ${ }^{\mathrm{ref}} \mathrm{F}_{\text {finger }}\left(=\boldsymbol{g}^{T} \sum_{i=1}^{5}{ }^{\text {ref }} \boldsymbol{f}_{i \text {-th finger }}\right)$, where $\boldsymbol{g}$ is the unit vector along gravitation, are evaluated. By using these parameters, the distance between the hand and the object, the presence or absence of contact between a fingertip and the object, the connection relation between the contacted object and the other object, and the grasping condition are evaluated and used in the segmentation tree. As a result, the motion data is segmented into primitive motions.

A new primitive motion that is required as part of a new task will be added by simple modification of the segmentation tree. Let us consider a turn-screw task as an example. This task is a new task, and it requires a turn motion around an axis. Hence, a part of the segmentation tree will be modified as shown in Fig. 5; that is, Turn will be added as a primitive task. Turn is a section in which the object is turned around an axis. This example shows that the segmentation tree is additive for a new primitive motion required in a new task.

\subsection{Task analysis}

A task's type is analyzed based on the sequence of the obtained primitive motions in the following manner: A sequence of primitive motions from Move to Release is one task, because the hand must move to the target object to do something at first and must release the object at the end of the task. When Insert is found in the sequence of primitive motions, it is a peg-in-hole task. When Pullout is found in the sequence of primitive tasks, it is a peg-pullout-from-hole task. When Turn is found in the sequence of primitive tasks, it is a turn-screw task. The other is a pick-and-place task. After task recognition, primitive motions are re-labeled based on the recognized task. For example, Push between Place and Release is combined as Place because Push was a fluctuation by the operator, and Insert and Pullout in a turn-screw task are combined as Turn because they happen at a low angular velocity of the target object. This modification process will be added with propriety when a new primitive motion is added. After the re-labeling based on the recognized task, the desired trajectories, contact points, and contact forces are analyzed within each segment. 


\section{Manipulability of Robot Hand}

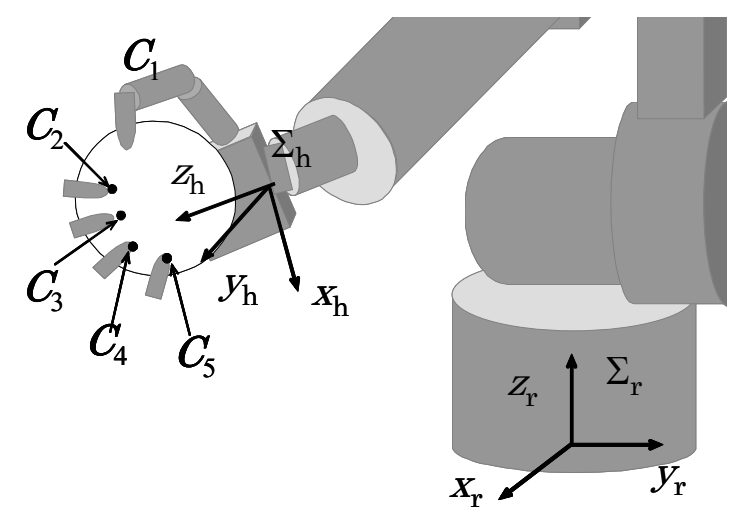

Fig. 6 Robot hand grasping an object

The geometrical form of the Gifu Hand III is similar to that of the human hand, but the two are not identical. In particular, the space between the thumb and opposing fingers is smaller in the Gifu Hand III than in the human hand, because of a mechanical design limitation. It follows that, when we use the human-hand model as a virtual hand, the teaching data of the fingertip positions include points that the robot fingers cannot attain. Even if the robot can grasp an object, it may not be able to grasp the object at the force taught to it. To solve this problem, we propose a virtual teaching method based on hand manipulability. In this method, the position and orientation of the robot hand, $\boldsymbol{r}_{\text {hand }}$, are determined so as to maximize the hand's manipulability on the condition that the robot grasps the object at the teaching contact points $\mathrm{C}_{i}$, as shown in Fig. 6. These points are variable for the stability of object grasping because they are based on the operator's skill. The manipulability measure of the robot hand is given by

$$
P I=\sum_{i=1}^{5}\left(w_{i} \sqrt{\left|\boldsymbol{J}_{i} \boldsymbol{J}_{i}^{T}\right|}+\rho_{i} P_{i}\right)
$$

where $w_{i}$ is a weighting coefficient of the $i$-th finger, $\boldsymbol{J}_{i}$ is a geometrical Jacobian of the $i$-th finger with respect to the hand coordinate frame, $P_{i}$ is an $i$-th penalty function to keep the joint angle in a movable range, and $\rho_{i}$ is a weighting coefficient. These satisfy the following relation for fingers:

$$
{ }^{\text {hand }} \boldsymbol{v}_{i \text {-th finger }}=\boldsymbol{J}_{i} \dot{\boldsymbol{q}}_{i-\text { th finger }}
$$

as well as the following relation for the thumb:

$$
\left[\begin{array}{c}
\boldsymbol{v}_{\text {stringer }} \\
\dot{\theta}_{1}
\end{array}\right]=\boldsymbol{J}_{1 \text { st finger }} \dot{\boldsymbol{q}}_{1 \text { st finger }}
$$

where hand $\boldsymbol{v}_{i \text {-th finger }}$ is the $i$-th finger velocity with respect to the hand coordinate, $\boldsymbol{q}_{i \text {-th finger }}=$ $\left[q_{1}{ }^{i}, q_{2}{ }^{i}, q_{3}{ }^{i}\right]^{\mathrm{T}}(1<i)$ is the $i$-th finger joint angle vector, $\boldsymbol{q}_{1 \text { st finger }}=\left[q_{1}{ }^{1}, q_{2}{ }^{1}, q_{3}{ }^{1}, q_{4}{ }^{1}\right]^{\mathrm{T}}$ is the thumb joint angle vector, and $\theta_{1}=q_{2}{ }^{1}+q_{3}{ }^{1}+q_{4}{ }^{1}$ is the angle between the thumb and the hand plate. The penalty function $P_{i}$ is given by

$$
P_{i}=-\sum_{j}\left(\frac{1}{\left(q_{j}^{i}-a_{j}^{i}\right)^{2}}+\frac{1}{\left(b_{j}^{i}-q_{j}^{i}\right)^{2}}\right),
$$

where $a_{j}^{i}$ and $b_{j}^{i}$ are the limit angles of the $j$-th joint of the $i$-th finger. The penalty function is added to the manipulability measure such that a solution of the $j$-th joint of the $i$-th finger exists in a movable range $a_{j}^{i} \leq q_{j}^{i} \leq b_{j}^{i}$. The geometrical Jacobian and penalty functions are rewritten in the position and orientation of the robot hand and the angle $\theta_{1}$ by using inverse kinematics of the fingers.

The manipulability measure is maximized by the method of steepest decent. In this method, the initial value of the robot hand position and orientation should be in the movable space. If the teaching position and orientation are not in movable space, an initial value 
search of the position and orientation of the robot hand is executed as a pre-processing. In the initial value search, the neighborhood space of the teaching position and orientation of the robot hand is decomposed into small subspaces, and the system examines whether a position and orientation in the decomposed subspace are in the motion space of the robot hand. If they are in the movable space of the robot hand, they become the initial value, thus completing the initial value search. This approach is time-consuming. When a human hand model in a virtual environment is scaled to the size of the robot hand model, this time-consuming process will not be needed. This is shown by the following experiment.

\section{Generation of the desired trajectory}

The differences in the profiles of multiple human motions may be important because they show the skill of the operator. Unnecessary motion may occur during the operator's demonstration. Although human motion is not always smooth, smooth motion is desirable in order to avoid exciting the robot's high-order vibration mode. The calculation time of the optimized motion trajectories must be shortened. The volume of data sent to the remote robot should be reduced. Considering these facts, the motion trajectory in the segment is approximated by a piecewise 5-degree polynomial in time, where the interval is divided by a starting point, extremal points and an endpoint of velocity. A bell-shaped velocity curve with one extreme is approximated by 2 polynomials, in which the boundary conditions at the connection point are such that the positions and velocities of the 2 polynomials are equal and the acceleration is zero. Hence, the number of intervals increases in proportion to the number of extremal points.

\section{Experiments}

\subsection{Hand model}

The solvability of optimum manipulability measurement depends on the hand position and orientation because the inverse kinematics problem of robot hand should be solved. Hence, searching for the initial position and orientation is required as pre-processing. If the virtual hand size is scaled to the size of the robot hand, it is expected to reduce the amount of pre-processing required. In this section, the effectiveness of scaling to the size of the robot hand is shown.

A task requiring the assembly of three pieces (sphere: diameter $20[\mathrm{~mm}]$, mass $0.2[\mathrm{~kg}]$, cylinder: diameter $40[\mathrm{~mm}]$, height $200[\mathrm{~mm}]$, mass $0.2[\mathrm{~kg}]$, and rectangular: mass 0.2 [kg], height $200[\mathrm{~mm}]$, width $40[\mathrm{~mm}]$, depth $40[\mathrm{~mm}]$ ) was examined by using two hand models: a human hand model at human hand size and a human hand model scaled to the robot hand size. The human motions were converted into robot commands based on the motion intention analysis. At the conversion, the teaching position and orientation of the robot hand at the start point of each segment were computed to maximize the manipulability of the robot hand. This was done on the condition that the teaching contact points on the object were not changed, because those points were decided based on human skill in grasping an object. Table 1 shows the results of the solvability of optimum manipulability measurement. This shows that pre-processing was not needed in the human hand model scaled to the robot hand size but it was always needed in the human hand model with the

Table 1 Number of required pre-processing

\begin{tabular}{|c|c|c|c|}
\hline \multirow{2}{*}{ Human hand model } & \multicolumn{3}{|c|}{ Number of required pre-processing } \\
\cline { 2 - 4 } & Sphere & Cylinder & Rectangular \\
\hline Human hand size & $7.85 \times 10^{3}$ & $7.35 \times 10^{3}$ & $2.73 \times 10^{2}$ \\
\hline Robot hand size & 0 & 0 & 0 \\
\hline
\end{tabular}


human hand size. The operability of both models for the operator was almost the same. Hence, the hand model scaled to the robot hand size is better than the hand model with the human hand size in terms of processing time required.

\subsection{Assembly task}

An assembly task of three pieces was executed in a virtual environment as shown in Fig. 7. Object 1 is a cylinder with a hole whose outer diameter, inner diameter and length are 130,41 , and $40[\mathrm{~mm}]$, respectively. Object 2 is a cylinder whose upper side diameter, lower side diameter, and length are 40, 40, and 180 [mm], respectively. Object 3 is a cylinder with a hole whose outer diameter, inner diameter and length are 80, 41, and 40 $[\mathrm{mm}]$, respectively. The operator executes the virtual robot teaching in a virtual assembly environment as shown in Fig. 8. First, the operator executes a pick-and-place task to place object 1 at a specified point. Then, the operator executes a peg-in-hole task to insert object 2 into the hole of object 1 . Finally, the operator executes a peg-in-hole task to assemble object 3 and object 2. Fig. 9 shows the measured parameters, primitive motions obtained by the proposed segmentation, and the recognized task, which consists of a pick-and-place task and two peg-in-hole tasks. Points on parameter curving line show the timing to separate the primitive motions. For example, Move and Approach in the pick-and-place task are

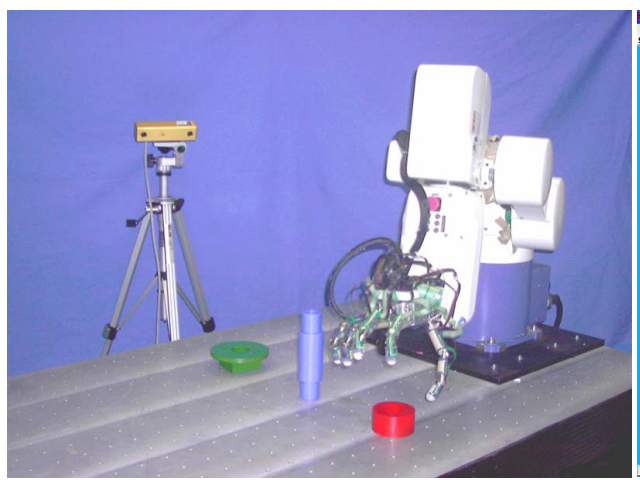

Fig. 7 Robot with Gifu Hand III and assemble work environment.

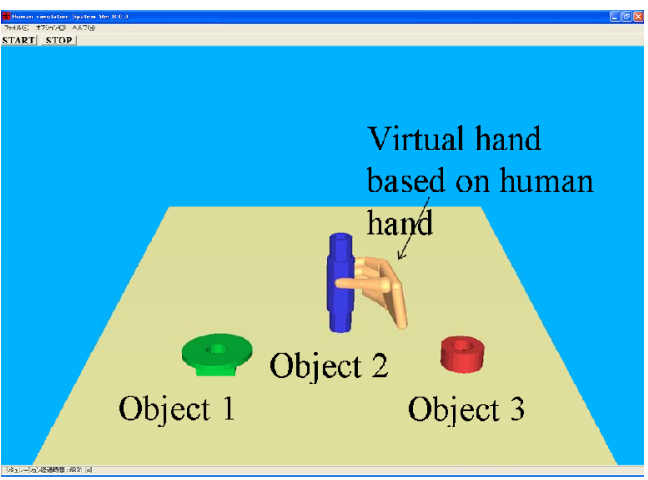

Fig. 8 Virtual assembly environment.

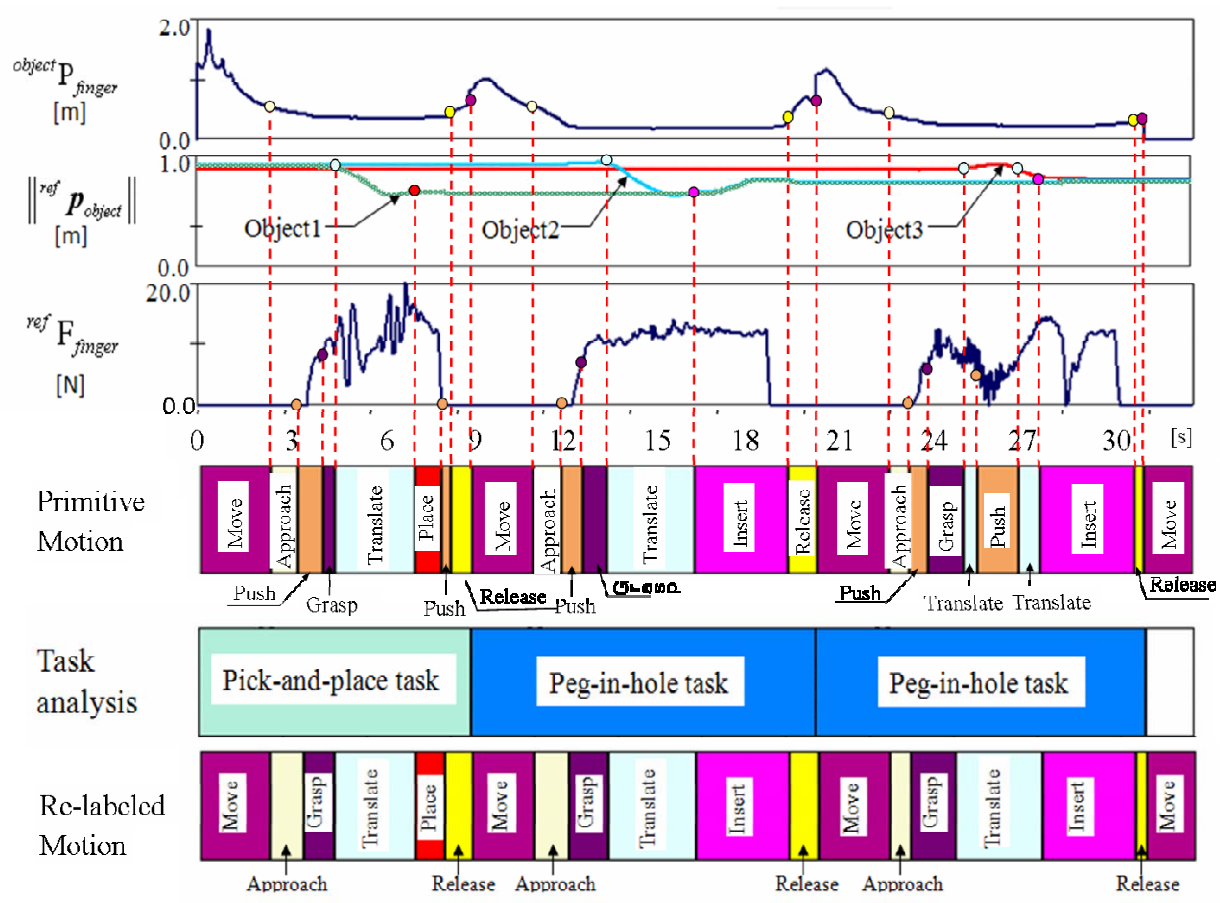

Fig. 9 Profiles of motion data and task analysis 
segmented by the magnitude of ${ }^{\text {object }} \mathrm{P}_{\text {finger }}$, and Grasp is segmented by the magnitude of ${ }^{\text {ref }} \mathrm{F}_{\text {finger. }}$ After the segmentation of primitive motions, a motion sequence from Move to Release is grouped into a task, and a task type is recognized. This shows that the segmentation of motion data and recognition of task are executed with propriety. After the recognition of task type, the primitive motions are re-labeled based on the task understanding. For example, Push between Approach and Grasp in the first peg-in-hall task is combined as Grasp because the Push was a fluctuation by the operator. Similarly, the second Push between Place and Release in the peg-in-hole is combined as Release. After re-labeling, robot finger trajectories are generated smoothly as described in section 4. After smoothing, the position and orientation of the robot hand is decided to maximize the hand manipulability measure. In this case, the hand manipulability measure increased $18 \%$ from that of the original teaching data. Using these smoothed and optimized desired trajectories, the robot motion was tested in the robot simulation system shown in Fig. 10(2). Finally, the tasks were executed experimentally by the 6 DOF robot arm with Gifu Hand III as shown in Fig. 10(3). These images show that the proposed segmentation can be applied to robot teaching including performance of plural tasks.

\section{Conclusions}

A new segmentation method for human motion analysis in VR robot teaching has been presented. The segmentation is executed according to a segmentation tree, in which the object position, fingertip position, presence or absence of contacts between the hand and a virtual object, reaction force at fingertip, and object position are evaluated. A task's type is analyzed based on the obtained sequence of primitive motions, and the primitive motions are re-labeled based on the recognized task. This method permits us to demonstrate plural tasks sequentially in a virtual reality environment. Secondly, in order to be able to map teaching data based on the human-hand model to teaching data for a robot hand, a virtual teaching method for multi-fingered robots based on a combination of scaling of the virtual hand model to the size of the robot hand and hand manipulability is presented. In this method, the position and orientation of the robot hand are determined so as to maximize the manipulability of the robot hand, on the condition that the robot grasps the object at the object's teaching contact point. This approach makes the virtual teaching system user-friendly. Our experimental results for performing an assembly task using a humanoid robot hand named Gifu Hand III demonstrate the effectiveness of proposed method.

\section{References}

(1) H. Asada and Y. Asari, "The Direct Teaching of Tool Manipulation Skills via the Impedance Identification of Human Motion," in Proc. IEEE Int. Conf. Robotics and Automation, pp.1269-1274, 1988.

(2) T. Kuniyoshi, M. Inaba, and H. Inoue, "Teaching by Showing: Generating Robot Programs by Visual Observation of Human Performance," in Proc. 20th Int. Symp. Ind. Rrobots, pp.119-126, 1989.

(3) K. Ikeuchi and T. Suehiro, "Toward an Assembly Plan from Observation - Part 1: Task Recognition with Polyhedral Objects," IEEE Trans. on Robotics and Automation, Vol. 10, No. 3, pp. 368-384, 1994.

(4) S. B. Kang and K. Ikeuchi, "Toward Automatic Robot Instruction from Perception-Temporal Segmentation of Tasks from Human Hand Motion," IEEE Trans. on Robotics and Automation, Vol. 11, No. 5, pp. 670-681, 1995.

(5) R. M. Voyles, J. D. Morrow, and P. K. Khosla, "Gesture-Based Programming for Robotics: Human-Augumented Software Adaptation," IEEE Intelligent Systems, November/ December, pp. 22-29, 1999. 


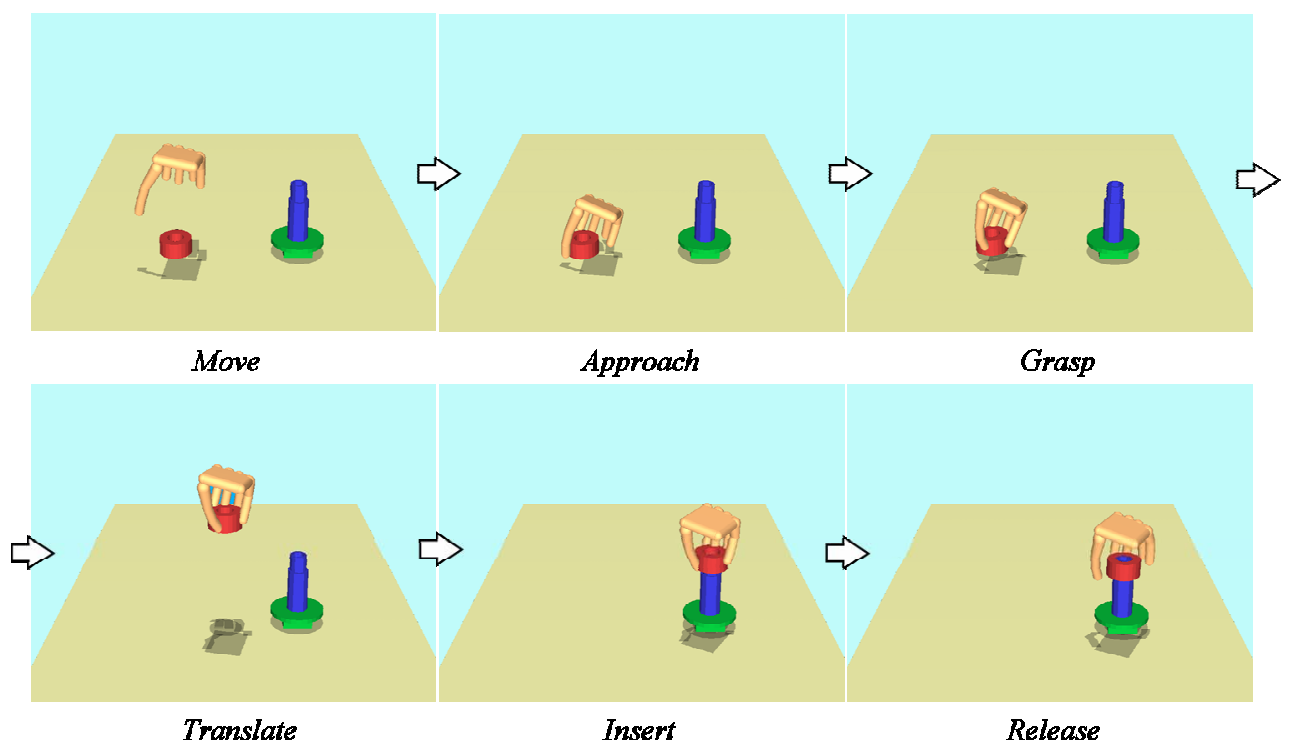

(1) Virtual robot teaching

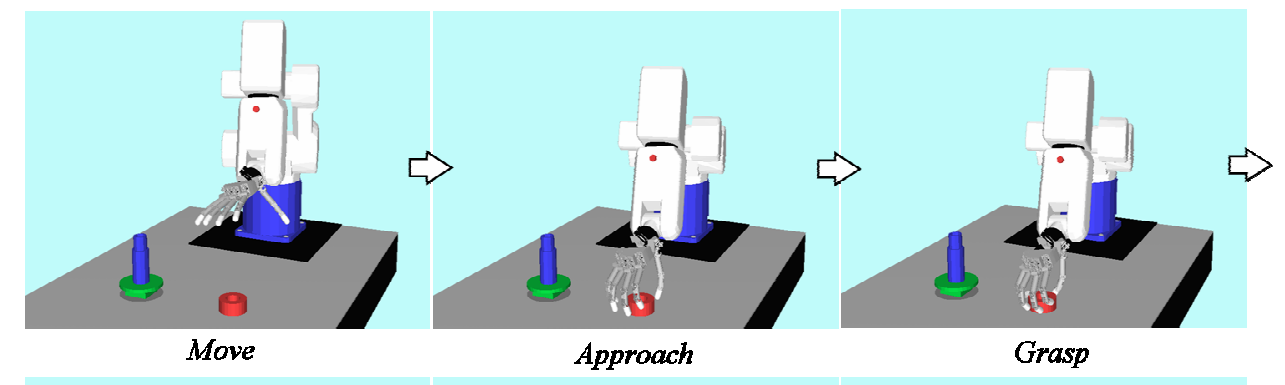

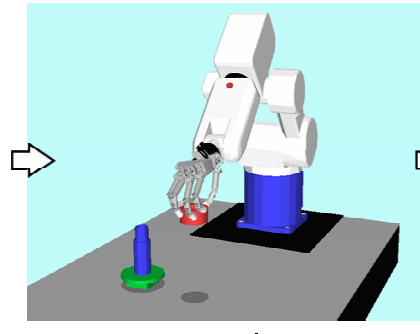

Translate

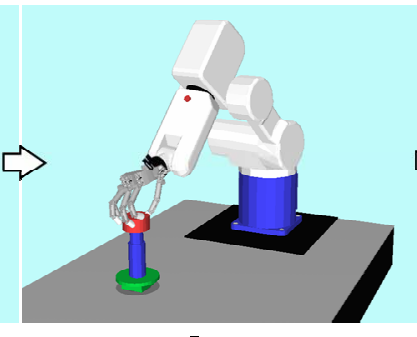

Insert

(2) Robot simulation

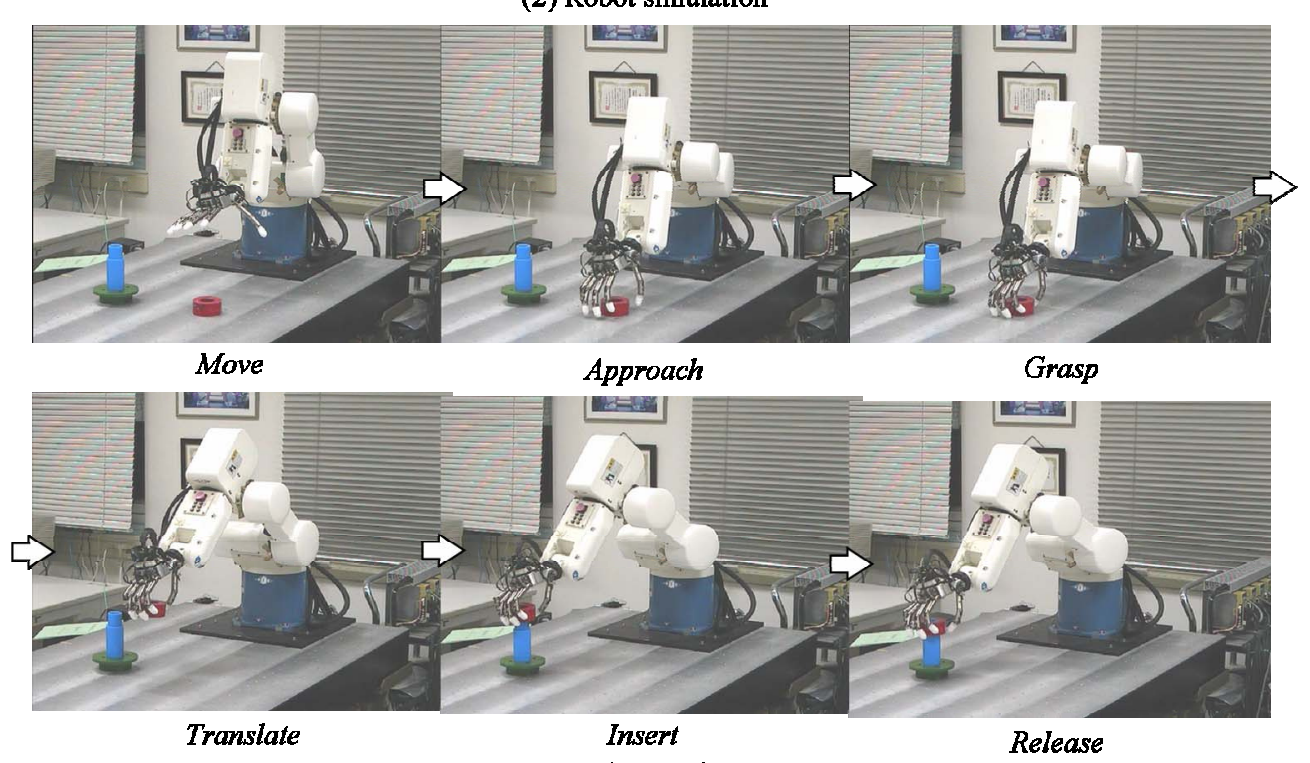

(3) Robot motion

Fig. 10 Peg-in-hole task 
(6) T. Takahashi, and H. Ogata, "Robotic Assembly Operation Based on Task-level Teaching in Virtual Reality," in Proc. IEEE Int. Conf. Robotics and Automation, pp. 1083-1088, 1992.

(7) T. Yoshikawa and K. Yoshimoto, "Haptic Virtual Reality Simulation of Assembly Operation," Jour. of TVRSJ, Vol. 4, No. 1, pp. 313-320, 1999. (in Japanese)

(8) M. Kaiser and R. Dillmann, "Building Elementary Robot Skills from Human Demonstration," in Proc. IEEE Int. Conf. Robotics and Automation, pp. 2700-2705, 1996.

(9) T. Sato, Y. Nishida, J. Ichikawa., Y. Hatamura, and H. Mizoguchi, “Active Understanding of Human Intention by a Robot through Monitoring of Human Behavior," Trans. of JRSJ, No. 4, Vol. 13, pp. 545-552, 1995. (in Japanese)

(10) M. Tsuda, H. Ogata, and Y. Nanjo, "Programming Groups of Local Models as a Model for Robotic Assembly from Human Demonstration,” in Proc. ICRA, pp.530-537, 1998.

(11) H. Kawasaki, K. Nakayama, and G. Parker, "Teaching for Multi-Fingered Robots Based on Intention of Motion in Virtual Reality," in Proc. IECON' 2000, pp.427-433, 2000.

(12) H. Kawasaki, K. Nakayama, and G. Parker, "Robot Teaching Based on Intention of Human Motion In Virtual Reality Environment," TVRSJ Vol. 5, No. 2, pp. 899-906, 2000. (in Japanese)

(13) H. Kawasaki, Kanji Nakayama, and Satoshi Ito, "On a Better Hand Model in Virtual Teaching for Multi-Fingered Robot," in Proc. VSMM2000, pp. 456- 464, 2000.

(14) S. B. Kang and K. Ikeuchi, "Toward Automatic Robot Instruction from Perception Mapping from Human Grasps to Manipulator Grasps," IEEE Trans. on Robotics and Automation, Vol. 13, No. 1, pp.81-95, 1997.

(15) R. N. Rohling and J. M. Hollerbach, "Optimized Fingertip Mapping for Teleoperation of Dexterous Robot Hand,” in Proc. IEEE Int. Conf. on Robotics and Automation, pp.769-775, 1993.

(16) T. Mouri, H. Kawasaki, K. Yoshikawa, J. Takai, and S. Ito, “Anthropomorphic Robot Hand: Gifu Hand III,” Proc. of Int. Conf. ICCAS2002, pp. 1288-1293, 2002

(17) T. Mouri, H. Kawasaki, and K. Umebayashi, "Developments of New Anthropomorphic Robot Hand and its Master Slave System,” Proc. of IROS 2005, pp. 3474-3479, 2005

(18) Virtual Technologies, Inc., "CyberGlove ${ }^{\mathbb{B}}$ User's Manual," 1997.

(19) M. R. Cutkosky, On Grasp Choice, Grasp Models, and the Design of Hands for Manufacturing Tasks, IEEE Trans. On Robotics and Automation, vol. 5, No. 3, pp. 269-279, 1989 